\title{
A User-centered Smartphone Application for Wireless EEG and its Role in Epilepsy
}

\author{
Sofía Ahufinger1,2*, Paloma Balugo³, María Mercedes González³, Elías Pequeño², Henar González ${ }^{4}$, Pilar \\ Herrero $^{1,2}$
}

${ }^{1}$ Ageing Lab, Centro de Tecnología Biomédica (CTB), Universidad Politécnica de Madrid (UPM) (Spain)

${ }^{2}$ Escuela Técnica Superior de Ingenieros Informáticos (ETSIINF), Universidad Politécnica de Madrid (UPM) (Spain)

${ }^{3}$ Servicio de Neurofisiología Clínica. Hospital Clínico San Carlos (HCSC) (Spain)

${ }^{4}$ Unidad de Innovación. Instituto de Investigación Sanitaria San Carlos (IdISSC) (Spain)

Received 18 March 2019 | Accepted 28 April 2019 | Published 22 June 2019

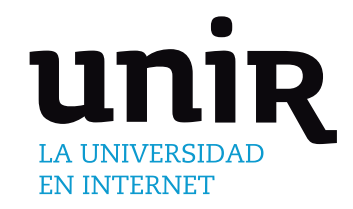

Electroencephalography is well-known for its importance in the diagnosis and treatment of mental and neurological disorders and abnormalities, being especially noted in critically ill patients who suffer a variety of cerebral injuries and altered states of consciousness. However, there is an important lack of adapted equipment and applications designed to suit the clinical and research needs. Hence, patients, physicians and researchers suffer, in most cases, from a restricted mobility due to non-portable devices and wires which keep them attached to the bed, leading to an uncomfortable patient experience or difficulties during the recording. In addition, nowadays, both physicians and researchers need to access the recordings and patient information from different places such as different units or hospitals. To solve this problem, this paper presents the design and evaluation of the high-fidelity prototype of a wireless EEG smartphone application based on a user-centred design, including expert panel guidance, paper and high-fidelity prototyping and usability testing, which confirm the accuracy of the defined context of use and the validity of the prototyped application to suit the clinical and research needs. In fact, since the EEG is the most efficient and specific way to define the epileptogenic cortex, we will focus on the possible use of the presented App in epilepsy diagnosis, which is one of the main targets in the field.

Electroencephalography, Epilepsy, Smartphone Application, Usercentered Design, Wireless.

\section{INTRODUCTION}

T LECTROENCEPHALOGRAPHY (EEG) plays a central role in

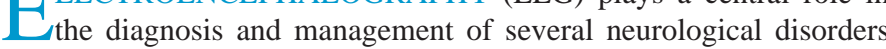
since its discovery in 1929 by Hans Berger [1]. Its role has been especially important for epilepsy since it is a convenient and relatively inexpensive way to detect physiological manifestations of abnormal cortical excitability [2]. The use of EEG is not only important for epilepsy diagnosis but for the location of epileptogenic zones and therapy evaluation [3]. In this case, the early diagnosis and management of the condition play an essential role when talking about overcoming seizure-related problems [4].

In fact, the use of continuous EEG monitoring is increasing in critical care units [5]. In an important number of cases, seizures are identified within the first 24 hours of monitoring [6] [7]. On the other hand, within the research field, EEG is being used to study and understand a variety of neurological disorders. Nevertheless, EEG requires highly qualified and expertise neurophysiologists, plus an extensive technical infrastructure. There is an important lack of equipment and applications designed to suit the clinical and research needs [8]. Hence, patients, physicians and

* Corresponding author.

E-mail address: sofia.ahufinger@ctb.upm.es researchers usually suffer from restricted mobility which is reflected in the recordings since it may lead to an uncomfortable patient experience or recording difficulties.

The user-centred design aims to provide a design and development methodology which involves end-users from the very beginning until the design validation [9]. This methodology includes expert panel guidance, paper and high-fidelity prototyping and usability testing, confirming and validating the defined context of use and the prototyped application. This approach is particularly interesting and important when talking about medical applications for two main reasons which are firstly, the strengthening of personalized medicine and secondly, that all the involved users -physicians, researchers and patients- need to focus on other important aspects rather than learning how to use a complex application, reason why some developed applications are not being used.

At last but not least, citizens nowadays require more and better healthcare services, promoting personalized medicine and transforming the health system model [10]. Information should be available from different locations such as different units within the same hospital or allowing the patient's monitoring from its own house [11].

\section{EPILEPSY}

Epilepsy is one of the most common and disabling neurologic disorders and can be defined as a heightened risk of suffering 
recurring episodes of seizures [12]. A seizure is an abnormal and hypersynchronous discharge of a group of neurons in the brain producing an altered neuronal function, induced by defects in cerebral tissues [13]. Furthermore, it is important to consider that isolated seizures or seizures which are directly related to acute brain aggression, such as drugs or head injury, are not considered as epilepsy. In fact, differentiating an epileptic seizure from a syncope is one of the main problems of this pathology.

The definition of epileptic syndrome refers to a group of clinical features and symptoms usually occurring together (e.g., types of seizures, patient age, precipitating factors).

Epileptic seizures are mainly classified as generalized and focal seizures. At the same time, generalized seizures can be divided into two depending on the presence or absence of convulsions. Focal seizures can be divided into three types: simple seizures (consciousness is retained), complex seizures (affecting the deepest zones of the temporal lobe) and secondarily generalized seizures.

Epilepsy diagnosis is essentially clinical as it is carried out mainly by evaluation of the detailed patient history, supported in most cases by findings in EEG since other clinical tests are often normal.

\section{A. Epilepsy in Children}

Although epilepsy has a higher occurrence in both extremes of life [14], during its development, the brain has a heightened susceptibility to seizures, which leads to an elevated number of epilepsy cases during childhood [15].

Nonetheless, performing EEGs to kids is not an easy task. Babies and kids tend to be lively, always in constant movement, increased by the medical environment, leading to two main problems.

This constant movement makes hard to keep the headset in the correct position and at the same time makes hard to perform the recording.

Then again, this may cause altered recordings that may lead to an impossible or wrong diagnosis. Hence, nurses and doctors often require entertaining them by using different toys and games. For example, balloons can often be found in neurophysiology units for children entertainment, producing static electricity when they are used, that is registered as a spike-and-wave pattern in EEG, which is a typical pattern found in epileptic seizures, causing a mistaken diagnosis if this problem is not detected.

\section{ELECTROENCEPHALOGRAPHY}

Electroencephalography allows measuring the electric activity of the brain using electrodes in different locations of the scalp. There are several diseases capable of mimicking seizure features, easily leading to a wrong diagnosis due to a mistaken clinical presentation [16]. Withal as we previously mentioned, EEG is the most specific noninvasive method to define the epileptogenic cortex, and hence, to diagnose epilepsy [17]. Nonetheless, its sensitivity and specificity are variable since they depend on several factors such as the procedure, montage or type of recording [18]. For this reason, both, positive and negative findings in EEG might not include or exclude the diagnosis of epilepsy respectively depending on the presence or absence of symptoms.

It is recommended to perform an EEG when the patient is suffering an apparently non-induced seizure, in order to determine wherever it is or not an epileptic seizure, type of seizure and predicting if the patient is going to suffer more episodes.

There are several types of EEG recordings, which are widely used for different purposes along the epilepsy diagnosis and monitoring. The basal EEG is performed while the patient is awake, with no need of previous preparation. The sleep-privation EEG is the same as the basal EEG but with a previous sleep-privation of 24 hours, to favour the correct patient sleep during the recording. Video-EEG provides video information synchronized with the EEG recording in order to provide information about the patient routine which can be important for the EEG interpretation. This last type of EEG is especially used in patients which are hard to diagnose or treat.

In adults with a first non-induced seizure, the first EEG shows epileptiform discharges not in all cases, but only in few of them. The probability of finding abnormalities increases when the EEG is performed during the first 48 hours since the first seizure. If the routine EEG does not detect any alteration, performing an EEG under sleepdeprived conditions increases the probability of detecting epileptiform discharges by an additional $13-35 \%$ of cases. If the diagnostic uncertainty persists, then a long-term video-EEG monitoring is considered when the epileptic events are frequent enough.

Nevertheless, the EEG technology has some limitations since the low signal amplitude $(1 \mu \mathrm{V}-200 \mu \mathrm{V})$ involves an important background noise, being easy for the electrodes to record a signal that was not generated by the brain or even by the body (e.g., device interferences). Hence, it is especially important to amplify and filter these signals in different ways in order to make possible its correct interpretation. Usually, EEG devices count with a high-pass filter (HPF) to delete low frequencies (e.g., eye movement) and a low-pass filter (LPF) to delete high-frequency signals (e.g., muscle movements).

\section{A. Montages}

The EEG system is based on the comparison of signals obtained by two different electrodes. Each line or EEG channel records the potential difference between two electrodes and amplifies it (derivation). The minimum number of electrodes that should be used for a normal EEG is 10 , however, in the case of epilepsy, there is a minimum number of 32 electrodes for allowing its correct diagnosis.

Montages should be as simple as possible but they must follow two rules which are: going from anterior to posterior locations and going from the left side to the right side of the scalp. Each combination of derivations is a different montage, being possible to use adjacent electrodes or distant ones, with two main types which are bipolar and monopolar montages.

In the bipolar montage, the difference between a pair of electrodes is amplified and recorded. This kind of montages can be anteriorposterior (sagittal) or transverse (coronal).

The monopolar montage, also known as referential montage, works comparing the signal obtained by a certain electrode with the one obtained by another electrode which is located in a reference zone with a lack of neural activity (e.g., the ear lobe), taking into consideration that if the reference electrode is located far from the location of the main electrode, the background noise of both electrodes would not be the same, possibly affecting the recording.

\section{10-20 System}

The 10-20 system, which name refers to the electrode location in the scalp, is the most used system for electrode location. For this system, both cranial bone protuberances (nasion and inion) are taken as references. Nasion is the zone when the forehead comes together with the nose, while inion refers to the external occipital protuberance.

To locate the electrodes, the distance between inion and nasion is measured. This measure should be divided into intervals of $10 \%$ and $20 \%$. The $10 \%$ of that distance measured from the nasion is where the fronto-polar (Fp) point should be located and the 10\% measured from the inion locates the Occipital (O) point. The frontal $(\mathrm{Fz})$, central $(\mathrm{Cz})$ and parietal $(\mathrm{Pz})$ points should be located between the $\mathrm{Fp}$ and $\mathrm{O}$ points (Fig. 1). The remaining electrodes are located following the same 
methodology, being possible to use a total amount of 22 electrodes. Both pre-auricular points are frequently used as ground references for the formed electrical circuit since both of them receive the same background noise but minimal neural activity.

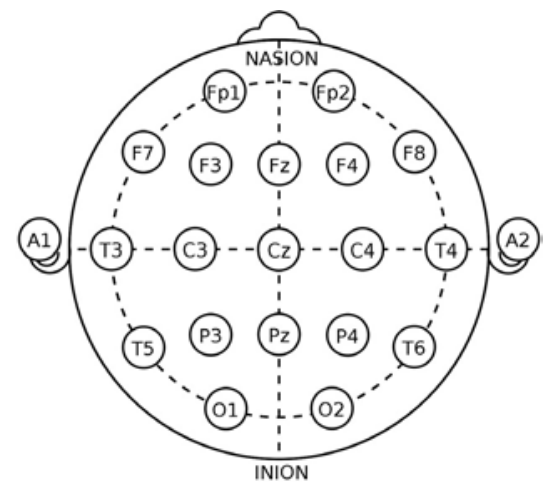

Fig. 1. International 10-20 system for electrode location in EEG procedures.

The head then is divided by the location of the electrodes, determining each one of the zones. Taking the middle line (z subindex) as the reference, even numbers should be located on the right side, while odd numbers should be located on the left.

\section{HARDWARE}

For the development of this project, we have followed a usercentred design which focuses on giving a solution to the needs and requirements specified by the end-users of the application. This group of needs and requirements are specified in the Context of Use document, which is defined after the observation and interviews of end-users. The different hardware parts were selected taking into account this Context of Use, in order to satisfy all needs and requirements of both, medical and research staff.

On the one hand, the OpenBCI [19] platform was selected because of the possibility of performing both hardware and software modifications freely, since it is the only platform providing a wireless and open-source solution for the human-computer interaction [20].

On the other hand, as previously mentioned, the epilepsy diagnosis requires an elevated number of channels, up to 24-32. This together with the need of real-time data streaming instead of saving data in a SD card, makes the Cyton board plus the Daisy module the best option.

The Cyton board offers 8 channels, with the addition of the Daisy module, the number of channels increases to 16 , which is not enough. To solve this problem, we proposed two different solutions.

The first option was modifying the Daisy module to include 3 ADS1299 chips, obtaining 32 channels when connected to the Cyton board that includes another ADS1299. This option was discarded due to the major modifications that the PCB would suffer, requiring the complete PCB redesign, unaffordable for its elevated cost in money and time and the high risk of a not working connection with the Cyton board.

The second and selected option was using two sets of Cyton board plus Daisy module, with 16 channels each of them. The main problem of this solution was synchronizing the two systems to obtain both samplings at the same time. Each Daisy module uses the clock signal of its own Cyton, and, in order to solve the previous issue, we decided to use the clock signal of one of the Cyton boards, in a way that both Cytons have the same sampling time and both Daisy modules follow the same clock signal.

The data obtained with these boards can be received by using the RFDuino included, but, this device cannot be used from a smartphone.
For this reason, we decided to obtain the recorded data using a WiFi shield connected to the Cyton and Daisy boards, obtaining at the same time a bigger bandwidth.

The headset, also from OpenBCI, is 3D printed following the 10-20 system, which means that there are specific places designed to locate the electrodes. The headset counts with 32 gaps for electrode location which can be used without any restriction and is available in different sizes. The headset will have both sets of boards and a battery attached, being completely wireless as seen in Fig. 2.

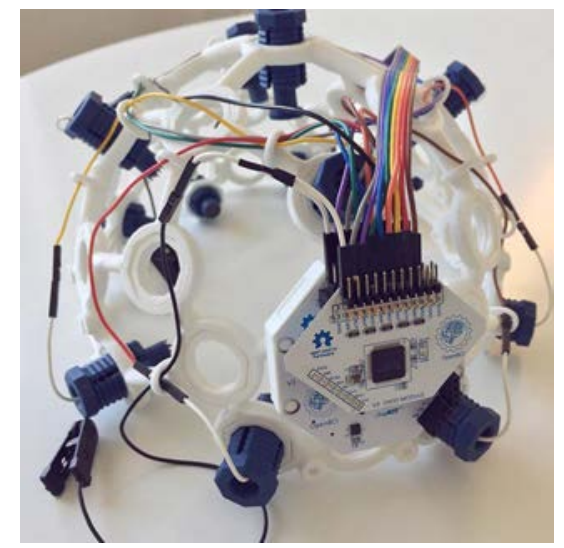

Fig. 2. OpenBCI wireless 3D-printed headset with the Cyton board and the Daisy module attached.

\section{IMPLEMENTATION}

The application interface and functionalities were designed following the Context of Use, just like the selection of hardware components. Firstly, a low-fidelity prototype was created and evaluated with different kinds of end-users, including doctors and researchers. All usability issues were found and corrected, and, all the suggested modifications and improvements were also included. Secondly, after the mentioned modifications, a high-fidelity prototype (Fig. 3) was created and evaluated by end-users, just like in the low-fidelity prototype case. After the evaluation, all the detected usability issues were again corrected, and the final features of the application were defined.

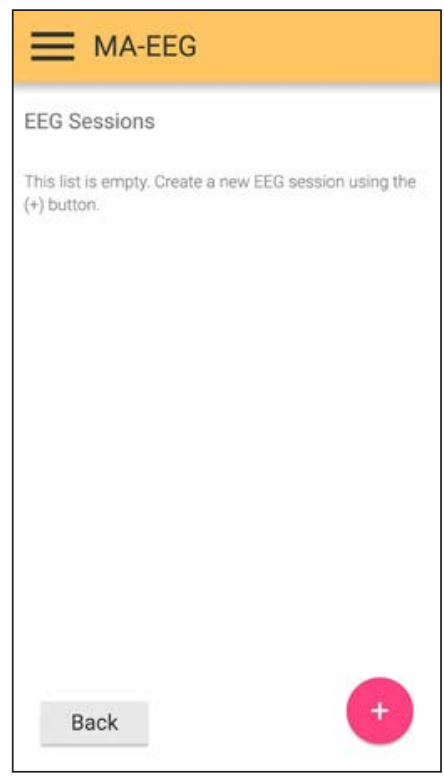

Fig. 3. Example screen obtained from the high-fidelity prototype of the application created for its evaluation. 
The mobile industry is growing each and every day, with over 5 billion smartphone users in the world by the end of 2022 [21]. Almost all of these smartphones run on three well-known platforms, which are Android, iOS and Windows mobile. For many years, the development of applications for each platform required specific tools and these applications could only run on a single platform, leading to a timeconsuming and expensive process since it required different specialized development teams for each platform.

During the last few years, the use of cross-platform mobile development has increased with the apparition of several frameworks like Xamarin or Flutter which allow developers to run the same code on different operating systems. However, cross-platform technologies allow for sharing $96 \%$ of the written code, requiring some platformspecific code if a native experience is wanted.

Xamarin (Microsoft) is one of the main cross-platform development technologies. This tool uses C\# as programming language and native platform libraries wrapped in a .NET layer, providing support for the three main platforms.

Alternatively, Flutter (Google), also open source, uses Google’s Dart as programming language and the $\mathrm{C}++$ engine, highlighting its good performance, but its applications are not portable to Windows mobile and 32-bit OS devices. In any case, this is not an important drawback in this study, since most of the used smartphones nowadays run on Android or iOS and the required processing power can only be supplied by relatively new smartphones.

About the programming language, Xamarin uses a widely used language which is C\#, instead of Dart like Flutter does. Despite this, although Dart is not as popular as Xamarin and its support community is still growing but quite limited at this point, it is very similar to Java and other OOP languages, making it easy to learn. For all the previously exposed reasons, the selected cross-platform framework for our project was Flutter.

\section{A. OpenBCI Simulator}

In order to develop the application without recording in real time with the headset, a board simulator was created. The WiFi shield acts just like an HTTP server, which is capable of sending the information to the smartphone or connected device. This shield responds to the Simple Service Discovery Protocol (SSDP) and hosts its unique name on local networks using multicast DNS (mDNS). To simulate this, we decided to simulate the board's REST service, creating an API web using .NET Core 2.1. For this purpose, we decided to use Swagger which is a language-agnostic specification for describing REST APIs, without any direct access to the implementation, minimizing the amount of work needed to connect disassociated services and document such service. Using the generated specification, the web-based user interface (UI) provided by Swagger offers information about the service (Fig. 4). This simulator provides the data that would be obtained by using a real OpenBCI board, offering data in two formats: JSON packets with Network Time Protocol (NTP) timestamp

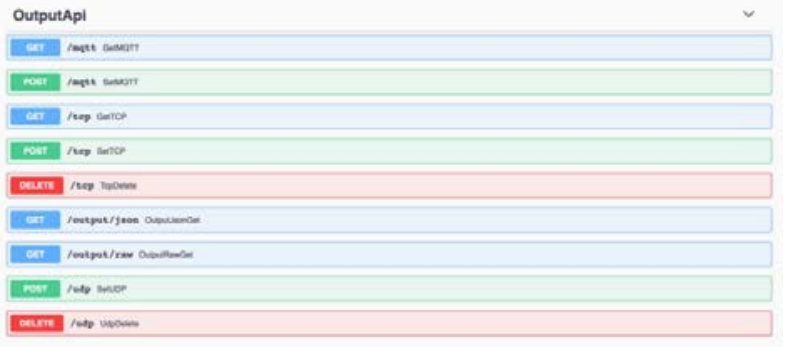

Fig. 4. OpenBCI WiFi shield REST service simulator in Swagger. The Swagger's UI provides information about each service, including the JSON and the raw data outputs.
In microseconds and arrays with channel data in nanovolts and 33byte raw packets, which is exactly the same format as the one used by the Cyton protocol.

\section{B. System Validation}

Before the final system establishment, the user-centered design defines the need for software testing and validation, being an important phase in the life cycle. The aim of the functional testing is to verify whether if each function of the application behaves in conformance with the specified requirements or not, considering the aim for which the software was designed.

This kind of tests provide higher stability and confidence about the system performance from the end-user point of view and not from the developer point of view. This is known as "black box" testing since it is not concerned about the source code, but about how the application behaves when being used for the defined test cases. Each functionality of the system is tested by providing appropriate input and verifying the type of output and its quality by comparing it with the expected results. The different aspects that can be evaluated are the mainline functions of the application, its basic usability and accessibility (previously evaluated with the low and high-fidelity prototypes) and the error conditions. It is important to consider that the effectivity of the functional testing increases when the test conditions are defined based on the requirements defined in the Context of Use.

The results of the functional tests showed some connectivity and signal issues related to the used hardware. Firstly, connectivity problems were observed during the data acquisition, stopping the recording a few times. Secondly, the obtained signal presented a high noise level making it difficult to analyze and to use the results for an accurate patient evaluation. These two problems have been related to the used hardware and its connectivity and signal quality. Finally, we found problems in the signal synchronization between the two 16-channel groups, due to the modifications that we had to perform in the PCBs in order to use one single clock signal for both Cyton+Daisy sets, also explaining the signal noise described previously.

\section{CONCLUSIONS AND FUTURE WORK}

Nowadays, more than ever, it is important to manage healthcare resources as efficiently as possible and this can only be done by creating and using new systems adapted to the current environment and needs. In this paper, we proposed a user-centred design process for the development of a wireless EEG that provides a useful and actualized tool adapted for the requirements of neurophysiologists and researchers in order to understand, diagnose and treat epilepsy.

Additionally, when the patient's freedom of movement is compromised, they tend to feel anxious and, therefore, their behaviour does not correspond to their usual routine. This situation makes hard to evaluate if seizures are related to any activity or specific moment during the patient's usual routine. We solved this problem with the OpenBCI wireless headset, offering a better patient experience, and also simplifying the procedure for medical and research staff, allowing patients to move freely.

Finally, the evaluation of both prototypes showed that the initial design met all the requirements exposed in the Context of Use, obtaining the final UI design and functionalities. All the found connectivity and noise issues will be solved during the development of the final version of the application, which is being implemented using the latest technology available, Flutter. It is expected to be used not only for the epilepsy diagnosis and treatment but also for Intensive Care Units monitoring and other medical or research applications where the patient's mobility plays a key role. 
ACKNOWLEDGMENT

This work has been supported by grants from the Community of Madrid (B2017/BMD-3760/NEUROCENTRO-CM) for research groups in Biomedicine, financed jointly by the European Social Found and European Regional Development Funds. In fact, Neurocenter (NEUROCENTRO-CM) is focused on the integrative study and treatment of neurological disorders.

\section{REFERENCES}

[1] S. Siuly, Y. Li, and Y. Zhang, "Significance of EEG Signals in Medical and Health Research,” Springer, Cham, 2016, pp. 23-41.

[2] S. J. M. Smith, "EEG in the diagnosis, classification, and management of patients with epilepsy,” J. Neurol. Neurosurg. Psychiatry, vol. 76, no. suppl_2, pp. ii2-ii7, 2005.

[3] J. Engel, “A practical guide for routine EEG studies in epilepsy.," J. Clin. Neurophysiol., vol. 1, no. 2, pp. 109-42, Apr. 1984.

[4] J. C. Das, "Electroencephalogram (EEG) in the management of epilepsy in children.," Mymensingh Med. J., vol. 23, no. 2, pp. 406-11, Apr. 2014.

[5] N. S. Abend, D. J. Dlugos, C. D. Hahn, L. J. Hirsch, and S. T. Herman, "Use of EEG Monitoring and Management of Non-Convulsive Seizures in Critically Ill Patients: A Survey of Neurologists,” Neurocrit. Care, vol. 12, no. 3, pp. 382-389, Jun. 2010.

[6] N. Jette, J. Claassen, R. G. Emerson, and L. J. Hirsch, "Frequency and Predictors of Nonconvulsive Seizures During Continuous Electroencephalographic Monitoring in Critically Ill Children,” Arch. Neurol., vol. 63, no. 12, p. 1750, Dec. 2006.

[7] K. Williams, R. Jarrar, and J. Buchhalter, "Continuous video-EEG monitoring in pediatric intensive care units," Epilepsia, vol. 52, no. 6, pp. 1130-1136, Jun. 2011.

[8] A. Jakab et al., "Novel wireless electroencephalography system with a minimal preparation time for use in emergencies and prehospital care," Biomed. Eng. Online, vol. 13, no. 1, p. 60, May 2014.

[9] A. D. V. DABBS et al., "User-Centered Design and Interactive Health Technologies for Patients,” CIN Comput. Informatics, Nurs., vol. 27, no. 3, pp. 175-183, May 2009.

[10] D. Rojas and J. Carnicero, "Big Data and Public Health Systems: Issues and Opportunities," Int. J. of Interact. Multimed. Artif. Intell., vol. 4, no. 7, pp. 53-59, 2018.

[11] A. Jalal, S. Kamal, and D. Kim, "A Depth Video-based Human Detection and Activity Recognition using Multi-features and Embedded Hidden Markov Models for Health Care Monitoring Systems," Int. J. of Interact. Multimed. Artif. Intell., vol. 4, no. 4, pp. 54-62, 2017.

[12] J. P. Karis and Expert Panel on Neurologic Imaging, "Epilepsy.,” AJNR. Am. J. Neuroradiol., vol. 29, no. 6, pp. 1222-4, Jun. 2008.

[13] P. A. Dekker, "Epilepsy A manual for Medical and Clinical Officers In Africa," 1998.

[14] V. Beletsky and S. M. Mirsattari, "Epilepsy, Mental Health Disorder, or Both?,” Epilepsy Res. Treat., vol. 2012, pp. 1-13, 2012.

[15] C. E. Stafstrom and L. Carmant, "Seizures and Epilepsy: An Overview for Neuroscientists," Cold Spring Harbor Perspectives in Medicine, vol. 5, no. 6, pp. a022426-a022426, June 2015.

[16] A. Tafakhori, V. Aghamollaii, S. Faghihi-Kashani, P. Sarraf, and L. Habibi, "Epileptic syndromes: From clinic to genetic.," Iran. J. Neurol., vol. 14, no. 1, pp. 1-7, Jan. 2015.

[17] M. Ercegovac and I. Berisavac, "Importance of EEG in intensive care unit,” Clin. Neurophysiol., vol. 126, no. 9, pp. e178-e179, Sep. 2015.

[18] S. Noachtar and J. Rémi, "The role of EEG in epilepsy: A critical review," Epilepsy Behav., vol. 15, no. 1, pp. 22-33, 2009.

[19] P. J. Durka, R. Kuś, J. Żygierewicz, and M. Michalska, "User-centered design of brain-computer interfaces : OpenBCI . pl and BCI Appliance," vol. 60, no. 3, pp. 427-431, 2012.

[20] A. Vourvopoulos and S. B. I. Badia, "Usability and Cost-effectiveness in Brain-Computer Interaction: Is it User Throughput or Technology Related?," in Proceedings of the 7th Augmented Human International Conference 2016 on - AH '16, Geneva, Switzerland, 2016, pp. 1-8.

[21] "Smartphone penetration worldwide 2014-2021 | Statista." [Online]. Available: https://www.statista.com/statistics/203734/global-smartphonepenetration-per-capita-since-2005/. [Accessed: 26-Feb-2019].

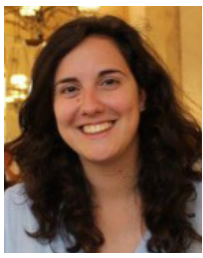

\section{Sofía Ahufinger}

She is a researcher at Centro de Tecnología Biomédica from Universidad Politécnica de Madrid. She received her degree in Health Engineering, major in Biomedical Engineering from Universidad de Málaga in 2017 and her Master Degree in Translational Research and Personalized Medicine from Universidad de Granada in 2018. She is preparing her $\mathrm{PhD}$ in Software, Systems and Computing at Universidad Politécnica de Madrid. In the research field, she is currently developing usercentered applications for diagnosis and treatment of several neurological disorders and visualization and analysis of brain connectivity.

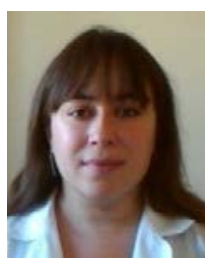

\section{Paloma Balugo}

She is a Consultant in Clinical Neurophysiology. She received her MD from Universidad Complutense, Madrid, Spain and finished her specialist training in Clinical Neurophysiology from Hospital Clínico San Carlos, Madrid, Spain. She has a Master Degree in Sleep Disorders and is pursuing a Master Degree in Epilepsy. She is a member of Spanish Society for Clinical Neurophysiology. She is currently heading the Electroencefalography and Evoked Potential areas of Clinical Neurophysiology Department, Hospital Clínico San Carlos, Madrid, Spain. Her area of clinical and research interest includes electrodiagnosis of epilepsy and coma.

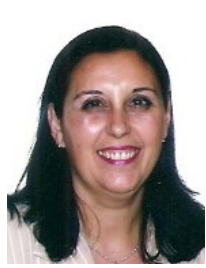

\section{María Mercedes González}

Dra. María de las Mercedes González Hidalgo, Graduate in Medicine and Medical Doctor by Complutense University (Madrid), Specialist Qualification in Clinical Neurophysiology in Clínica Puerta de Hierro (Madrid), Medicine Department assistant professor (Neuroloy Chair) of School of Medicine at the Complutense University (Madrid). Currently, head of Clinical Neurophysiology department of Hospital Clínico san Carlos in Madrid.

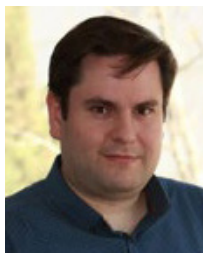

\section{Elías Pequeño}

B.S. in Computer Science from Universidad Politécnica de Madrid (UPM) with 13 years of experience in professional consulting services and implementation of Microsoft Dynamics ERP and CRM enterprise solutions. Currently studying a Ph.D. in Software, Systems and Computation at the UPM while working as CTO at Goom Spain, where he guides the design and development of customized business solutions and the architecture of systems and integrations based on Microsoft Azure cloud services.

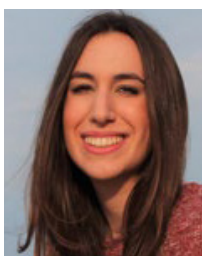

\section{Henar González}

She is a Biomedical Engineer from Universidad Politécnica de Madrid. She is currently working at the Innovation Unit \& SmartHealth Lab of the Health Research Institute of Hospital Clínico San Carlos. She works on the designing and implementation of clinical decision support systems and on the improvement of the efficiency of healthcare processes within the organization. During her MSc in Management and Innovation in Healthcare Technologies, she combined an internship in the MIT Regional Entrepreneurship Acceleration Program, which was aimed to foster Madrid entrepreneurial ecosystem, with the study of Open Innovation strategies in Life Sciences corporations. She is particularly interested in encouraging the engagement of women in technical careers, and thus has participated as a speaker in different initiatives such as WomenTechMakers.

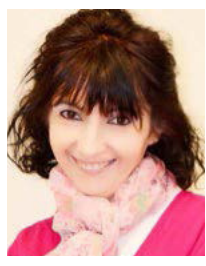

Pilar Herrero

She is an Associate Professor at the Universidad Politécnica de Madrid (Spain). Author of more than 80 peer-reviewed international publications and editor of more than 15 international publications, European Ph.D. in Computer Science and extraordinary Ph.D. Award, in the last few years, Prof. Herrero has been involved in the organization of more than a hundred international events. She has also been involved in more than one hundred and fifty International Program Committees. 